\title{
Estimação de Parâmetros de um Modelo Semi-Empírico de Transferência Simultânea de Calor e Água no Solo
}

\author{
Emanueli Bandeira Avi, $\quad$ Peterson Cleyton Avi, \\ Depto de Ciências Exatas e Engenharias, DCEEng, UNIJUI \\ 98700-000, Ijui, RS \\ E-mail: emanueli.b@hotmail.com; peterson.avi@unijui.edu.br
}

\author{
Pedro Augusto Pereira Borges \\ Universidade Federal da Fronteira Sul - UFFS \\ 89812-000, Centro, Chapecó, SC \\ E-mail: papborges@gmail.com
}

\begin{abstract}
Resumo: No solo, o calor e água são importantes na germinação de sementes, no desenvolvimento de plantas e no transporte de nutrientes e solutos. Sendo assim, é proposto um modelo semi-empírico com acoplamento dos problemas hidráulico e térmico no solo simulado através de dados sintéticos. O modelo é composto por um sistema de duas Equações Diferenciais Parciais: a Equação de Richards e a Equação da Energia. Neste trabalho, o Problema Inverso foi utilizado para estimar parâmetros com base em dados sintéticos e verificar se o modelo semi-empírico proposto é viável do ponto de vista de tempo computacional para ser utilizado como Problema Direto de um Problema Inverso. Devido ao grande número de parâmetros a serem determinados foi utilizado o Método de LevenbergMarquardt, que se mostrou eficiente quanto à precisão e tempo computacional. A eficiência deste método está diretamente ligada ao parâmetro de regularização utilizado no mesmo.
\end{abstract}

PALAVRAS-CHAVE: Modelo semi-empírico, Problema Inverso, Método de LevenbergMarquardt.

\section{INTRODUÇÃO}

A agricultura é uma atividade que gera alimentos e riquezas. O estudo do solo e suas propriedades além de ser indispensável para o desenvolvimento da agricultura também abrange estudos importantes para o desenvolvimento de obras de engenharia civil, bem como o entendimento do ecossistema. No solo, os gradientes de temperatura e o teor de água são importantes na germinação de sementes, no desenvolvimento de plantas e no transporte de nutrientes e solutos.

AVI (2011) propôs um modelo semi-empírico, que consiste no acoplamento dos problemas hidráulico e térmico através de dados experimentais. Devido à indisponibilidade de dados experimentais de laboratório ou de campo foram realizadas simulações utilizando dados sintéticos (artificiais) supondo o comportamento das variáveis.

O modelo semi-empírico consiste na resolução do sistema de Equações Diferenciais Parciais composto pela equação que descreve a dinâmica da água no solo (Equação de Richards) e pela equação que descreve o comportamento da temperatura no solo (Equação do Calor). O acoplamento é justificado por Hillel (1998) que afirma que gradientes de temperatura induzem a transferência de água, assim como, gradientes de teor de água induzem a transferência de calor. Essa reciprocidade nos sugere que as propriedades hidráulicas sofrem influência da temperatura e as propriedades térmicas sofrem influência da dinâmica da água no solo.

Neste trabalho, o Problema Inverso foi utilizado para: analisar o desempenho do modelo semi-empírico proposto para a determinação dos parâmetros; bem como, para a análise do método utilizado em relação a precisão na determinação dos parâmetros e a economia do tempo de simulação.

Quando se objetiva analisar os efeitos resultantes a partir do conhecimento das causas, trata-se de um Problema Direto. Já no Problema Inverso deseja-se conhecer as causas, através 
de observações dos efeitos que são conhecidos. Entende-se por causas, num modelo matemático, as condições iniciais e de contorno das equações diferenciais parciais, termos de fonte e propriedades do sistema e/ou material (VELHO, 2008).

Foi utilizado o Método de Levenberg-Marquardt (MLM) (OZISIK e ORLANDE, 2000). A opção por este método se deve ao grande número de parâmetros a serem determinados. Este é um método determinístico, assim como o Método de Newton e o Método de Quase-Newton. O MLM apresenta ótimas taxas de convergência devido ao parâmetro de regularização, que é determinado através de testes numéricos, sendo isto o que diferencia este método dos outros dois citados.

\section{METODOLOGIA}

\section{Problema Direto}

Nas simulações do problema Inverso, o modelo semi-empírico caracteriza o problema Direto, que consiste na resolução simultânea do sistema de Equações Diferenciais Parciais formado pela Equação de Richards, que por sua vez descreve a dinâmica da água no solo, dado pela Equação (1), com suas respectivas condições de contorno e inicial, (Eqs. (2), (3) e (4)):

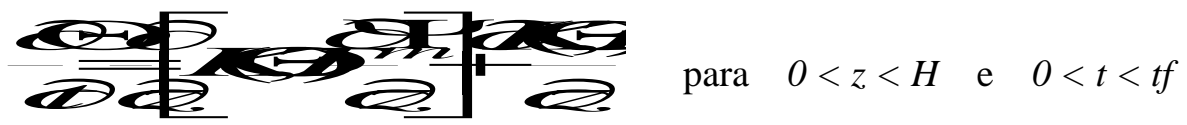

$\Theta(Q)=\Theta_{1}(t)$ para $0<t<t f$

$$
\frac{\partial \Theta}{\partial z}(\boldsymbol{H} \boldsymbol{t})=\mathrm{C} \quad \text { para } \quad 0<t<t f
$$

$$
\Theta(z, 0)=\Theta(z) \text { para } 0<z<H
$$

Onde: $\Theta$ é o teor de água (adimensional); $K$ é a condutividade hidráulica $(\mathrm{m} / \mathrm{s}) ; \Psi_{m}$ é o potencial matricial $(\mathrm{Pa}) ; z$ é a profundidade $(\mathrm{m}) ; \mathrm{H}$ é a altura da coluna de solo $(\mathrm{m}) ; t$ é o tempo $(s)$; tf é o tempo final $(s) ; T$ é a temperatura $\left({ }^{\circ} \mathrm{C}\right) ; \Theta_{1}$ é a condição de contorno para a superfície (adimensional); $\Theta_{0}$ é a condição inicial (adimensional). Juntamente com a Equação do Calor, que descreve o comportamento da temperatura no solo, dado pela Equação (5), com suas respectivas condições de contorno e inicial, (Eqs. (6), (7) e (8)):

$$
\begin{aligned}
& \frac{D}{\partial 2} \text { para } 0<z<H \text { e } 0<t<t f \\
& T(0, t)=T_{2}(t) \quad \text { para } 0<t<t f \\
& T(H, t)=T_{1}(t) \quad \text { para } \quad 0<t<t f \\
& \mathbf{T ( z ,},=\boldsymbol{T}_{\mathbf{O}}(z) \quad \text { para } \quad 0<z<H
\end{aligned}
$$

Onde: $\alpha$ é a difusividade térmica $\left(\mathrm{m}^{2} / \mathrm{s}\right) ; T_{2}$ e $T_{1}$ são, respectivamente, as condições de contorno para a superfície e fundo $\left({ }^{\circ} \mathrm{C}\right)$; $T_{0}$ é a condição inicial $\left({ }^{\circ} \mathrm{C}\right)$.

O acoplamento ocorre pela consideração da variação da condutividade hidráulica saturada em função da temperatura, Equação (9), que vem a contribuir para determinar a condutividade 
hidráulica utilizada na Equação de Richards dada por Van Genuchten (1980), conforme Equação (10).

$$
\begin{aligned}
& K_{o}=\left(c_{1} \cdot T+c_{2}\right) \cdot 2.7 \cdot 10^{-6} \\
& K(\Theta, T)=K_{0} \Theta^{l}\left[1-\left(1-\Theta^{1 / m}\right)^{m}\right]^{2}
\end{aligned}
$$

Onde: $c_{1}, c_{2}$ são parâmetros de ajuste a serem determinados; $K_{0}$ é a condutividade hidráulica saturada $(\mathrm{m} / \mathrm{s})$. Simultaneamente, também é considerado a variação da difusividade térmica, utilizada na Equação do Calor, em função do teor de água, conforme Equação (11).

$$
\alpha(\Theta)=\left(c_{3} \cdot e^{\left(c_{4} \cdot \Theta\right)}+c_{5}\right) \cdot 10^{-7}
$$

Onde: $c_{3}, c_{4}, c_{5}$ são parâmetros de ajuste a serem determinados.

Essas dependências se obtidas de forma empírica, são o que caracterizam o modelo semiempírico, porém nas simulações foram utilizados dados sintéticos. O sistema de equações do modelo foi resolvido numericamente pelo Método de Diferenças Finitas, utilizando o esquema temporal Explícito Simples.

Foi desenvolvido um algoritmo utilizado para o cálculo da distribuição de temperatura e teor de água em cada posição na coluna de solo e tempo. $\mathrm{O}$ algoritmo foi implementado no software MATLAB.

\section{Problema Inverso}

O MLM foi utilizado para determinar os coeficientes $a, m, n$ da equação característica do solo e os parâmetros das funções de dependências assumidas a partir do modelo semi-empírico (Problema Direto). Para tanto, foi necessário determinar separadamente os parâmetros de cada problema, sendo criados dois vetores de parâmetros. No vetor do problema hidráulico $(X \Theta)$, tem-se os parâmetros $c_{1}$ e $c_{2}$ da Eq. (9) e os coeficientes $a$ e $n$ da curva característica do solo, assumindo que $\boldsymbol{m}=\mathbf{1}-1 / \boldsymbol{n}$ de acordo com VAN GENUCHTEN (1980), portanto, $X \Theta=\left[c_{1} ; c_{2} ; a ; n\right]$. No vetor do problema térmico $(X T)$, tem-se os parâmetros $c_{3}, c_{4}, c_{5}$ da Eq. (11), portanto, $X T=\left[c_{3} ; c_{4} ; c_{5}\right]$. Em seguida, é apresentado o algoritmo utilizado:

$1^{\circ}$ Passo: Resolver o Problema Direto: problema hidráulico $P D_{\Theta}$ e problema térmico $P D_{T} \quad$ com as estimativas iniciais

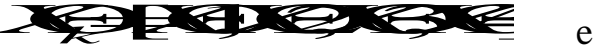
XFEMTRE

$2^{\circ}$ Passo: Calcular a soma dos erros quadrados ou função objetivo pela Eq. (12).

$$
\begin{aligned}
& S \Theta\left(X \Theta_{k}\right)=\left[Y \Theta-P D_{\Theta}\left(X \Theta_{k}\right)\right]^{T} \cdot\left[Y \Theta-P D_{\Theta}\left(X \Theta_{k}\right)\right] \\
& S T\left(X T_{k}\right)=\left[Y T-P D_{T}\left(X T_{k}\right)\right]^{T} \cdot\left[Y T-P D_{T}\left(X T_{k}\right)\right]
\end{aligned}
$$

onde

$Y \Theta$ e $Y T$ são os dados sintéticos.

$3^{\circ}$ Passo: Cálculo da matriz Jacobiana para ambos os casos. 


$$
J \Theta=\left[\begin{array}{cccc}
\frac{\partial P D_{\Theta 1}}{\partial X \Theta_{1}} & \frac{\partial P D_{\Theta 1}}{\partial \mathrm{X} \Theta_{2}} & \cdots & \frac{\partial P D_{\Theta 1}}{\partial \mathrm{X} \Theta_{4}} \\
\frac{\partial P D_{\Theta 2}}{\partial X \Theta_{1}} & \frac{\partial P D_{\Theta 2}}{\partial \mathrm{X} \Theta_{2}} & \cdots & \frac{\partial P D_{\Theta 2}}{\partial \mathrm{X} \Theta_{4}} \\
\cdot & \cdot & \cdot & \cdot \\
\cdot & \cdot & \cdot & \cdot \\
\cdot & \cdot & \cdot & \cdot \\
\frac{\partial P D_{\Theta \mathrm{n}}}{\partial \mathrm{X} \Theta_{1}} & \frac{\partial P D_{\Theta \mathrm{n}}}{\partial \mathrm{X} \Theta_{2}} & \cdots & \frac{\partial P D_{\Theta \mathrm{n}}}{\partial \mathrm{X} \Theta_{4}}
\end{array}\right] \quad J T=\left[\begin{array}{ccc}
\frac{\partial P D_{T 1}}{\partial X T_{1}} & \frac{\partial P D_{\mathrm{T} 1}}{\partial \mathrm{X} T_{2}} & \frac{\partial P D_{\mathrm{T} 1}}{\partial \mathrm{XT}} \\
\frac{\partial P D_{T 2}}{\partial X T_{1}} & \frac{\partial P D_{\mathrm{T} 2}}{\partial \mathrm{XT} T_{2}} & \frac{\partial P D_{\mathrm{T} 2}}{\partial \mathrm{X} T_{3}} \\
\cdot & \cdot & \cdot \\
\cdot & \cdot & \cdot \\
\frac{\partial P D_{\mathrm{Tn}}}{\partial \mathrm{X} T_{1}} & \frac{\partial P D_{\mathrm{Tn}}}{\partial \mathrm{XT}} & \frac{\partial P D_{\mathrm{Tn}}}{\partial \mathrm{X} T_{3}}
\end{array}\right]
$$

$4^{\circ}$ Passo: Cálculo da nova estimativa para $X \Theta_{k+1}$ e $X T_{k+1}$, através da Eq. (13)

$$
\begin{aligned}
& X \Theta_{k+1}=X \Theta_{k}+\left[(J \Theta)^{T} \cdot J \Theta+\mu_{k} \Omega_{k}\right]^{-1} \cdot\left[Y \Theta-P D_{\Theta}\left(X \Theta_{k}\right)\right] \\
& X T_{k+1}=X T_{k}+\left[(J T)^{T} \cdot J T+\mu_{k} \Omega_{k}\right]^{-1} \cdot\left[Y T-P D_{T}\left(X T_{k}\right)\right]
\end{aligned}
$$

onde: $\mu_{k}$ é um parâmetro de regularização e $\Omega_{k}$ é a matriz diagonal denominada termo de regularização.

5 Passo: Resolver o Problema Direto $P D_{\Theta}$ e $P D_{T}$ com a nova estimativa $X \Theta_{k+1}$ e $X T_{k+1}$.

$6^{\circ}$ Passo: Calcular $S \Theta\left(X \Theta_{k+1}\right)$ e $S T\left(X T_{k+1}\right)$ com a Eq. (12).

$7^{\circ}$ Passo: Se $X \mu_{k}$, onde $X$ é um numero real maior que 1. Retornar para o passo 4.

$8^{\circ}$ Passo: Se estimativa e substituir $\mu_{k}$ por $D \mu_{k}$, onde $D$ é um numero real menor que 1 e maior que 0 .

9० Passo: Verificar o critério de parada $S \Theta \leq \& \Theta$ e $S T \leq \& T$, se os dois critérios de parada forem satisfeitos interromper o processo e gerar a solução; caso contrário, substituir $k$ por $k+1$ e voltar ao passo 3 .

\section{RESULTADOS E DISCUSSÃO}

Na Figura 1 é apresentada a distribuição do teor de água e temperatura para todo o domínio espacial e $\mathrm{t}=50400 \mathrm{~s}$.
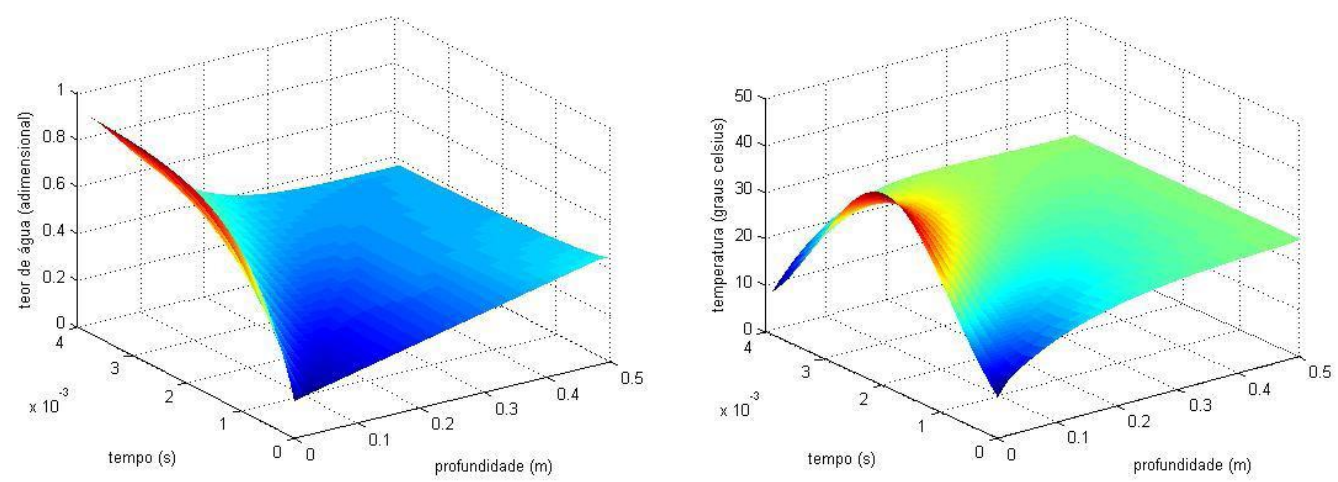

Figura 1 - Distribuição do teor de água e temperatura para $\mathrm{t}=50400 \mathrm{~s}$. 
$\mathrm{Na}$ superfície percebe-se um processo lento de irrigação, enquanto que na profundidade de $0,5 \mathrm{~m}$ o fluxo de água é considerado nulo. Os gradientes mais significativos de teor de água acontecem próximos a superfície, enquanto que para profundidades maiores os gradientes são mínimos. Em relação a temperatura percebe-se que na superfície o comportamento é de um dia de sol com poucas nuvens, onde a temperatura do solo aumenta até um ponto máximo e após passa a diminuir. $\mathrm{Na}$ profundidade de $0,5 \mathrm{~m}$ a temperatura é constante, sendo assim, os principais gradientes de temperatura ocorrem próximos a superfície, enquanto em profundidades maiores a temperatura sofre variação mínima durante o dia.

Em relação ao Problema Inverso, nas simulações foi variado o parâmetro de regularização $(\mu)$ para verificar a influência desse parâmetro no desempenho do método em relação à precisão na determinação dos parâmetros desejados e tempo computacional de simulação. Foi utilizada a seguinte estimativa inicial para verificar o comportamento do método: $X \Theta=[0,085 ; 15 ; 0,6 ; 15]$ e $X T=[15 ; 5 ; 10]$.

\begin{tabular}{|c|c|c|c|c|c|c|}
\hline$\mu$ & $\begin{array}{c}\text { Critério de } \\
\text { parada }\end{array}$ & Itera. & Tempo & $\begin{array}{c}\text { Parâmetros } \\
(\square)\end{array}$ & $\begin{array}{c}\text { Parâmetros } \\
(T)\end{array}$ & $R^{2}$ \\
\hline 0,05 & $\begin{array}{l}\& \Theta=10^{5} \\
\& T=10^{5}\end{array}$ & 8 & $2 \min 49 s$ & $\begin{array}{c}c_{1}=0,08 \\
c_{2}=21,352 \\
a=0,4 \\
n=13\end{array}$ & $\begin{array}{c}c_{3}=8,6911 \\
c_{4}=2,065 \\
c_{5}=12,185\end{array}$ & $\begin{array}{l}\Theta \cong 1 \\
T \cong 1\end{array}$ \\
\hline 0,45 & $\begin{array}{l}\& \Theta=10^{5} \\
\& T=10^{5}\end{array}$ & 18 & $10 \min 12 \mathrm{~s}$ & $\begin{array}{c}c_{1}=0,08 \\
c_{2}=21,352 \\
a=0,4 \\
n=13\end{array}$ & $\begin{array}{c}c_{3}=8,6912 \\
c_{4}=2,065 \\
c_{5}=12,184\end{array}$ & $\begin{array}{l}\Theta \cong 1 \\
T \cong 1\end{array}$ \\
\hline
\end{tabular}

Tabela 1 - Resultados dos testes numéricos do Método de Levenberg-Marquardt.

Os valores dos parâmetros sintéticos a serem determinados são $X \Theta=[0,08 ; 21,352 ; 0,4 ; 13]$ e $X T=[8,6911 ; 2,0644 ; 12,185]$. O Método de Levenberg-Marquardt, é um método eficiente na determinação de uma grande quantidade de parâmetros utilizando tempo computacional curto se comparado, por exemplo, com o Método de Procura em Rede Modificado. Essa eficiência no tempo computacional se deve ao parâmetro de regularização $(\mu)$ que acelera a convergência.

A Tabela 1 mostra a importância da análise do parâmetro de regularização $(\mu)$. Foram feitos testes numéricos exaustivos na procura de um valor eficiente para este parâmetro. Considerando a malha escolhida o valor eficiente encontrado para $(\mu)$ é 0,05 , tendo em vista que para valores menores a solução do Problema Direto é divergente.

O modelo semi-empírico proposto pode ser utilizado como Problema Direto em um Problema Inverso, sendo que a convergência na determinação de parâmetros acontece de forma satisfatória. Portanto, o modelo semi-empírico proposto é uma alternativa interessante na determinação acoplada da temperatura e teor de água do solo e na determinação de parâmetros específicos do solo como a difusividade térmica e a condutividade hidráulica. 


\section{CONCLUSÃO}

O Método de Levenberg-Marquardt se mostrou uma eficiente técnica na determinação de parâmetros quando em se tratando de um grande número de parâmetros se comparado com outros métodos como o de Procura em Rede Modificado. É um método determinístico, que neste caso, apesar de serem determinados sete parâmetros, tem um tempo de simulação computacional satisfatório. Muito dessa economia de tempo, em relação a outras técnicas de Problema Inverso, se deve ao parâmetro de regularização utilizado como um acelerador da convergência. É necessária uma análise através de testes numéricos para encontrar o valor eficiente desse parâmetro que pode resultar em ganho de tempo computacional.

O modelo semi-empírico representa uma alternativa eficiente para a simulação das transferências de calor e água no solo, desde que, sejam obtidas experimentalmente as dependências da condutividade hidráulica do solo saturado em função da temperatura e da difusividade térmica em função do teor de água. $\mathrm{O}$ acoplamento é evidente e deve ser utilizado para quantificar com melhor precisão a temperatura e o teor de água no solo, tornando as análises relacionadas ao solo mais significativas. A principal contribuição do modelo semiempírico está na determinação do teor de água, que é realizado geralmente com a equação de Richards desconsiderando a influência da temperatura. Com o modelo semi-empírico o teor de água é determinado tendo em vista essa influência da temperatura, tornando o valor mais preciso.

\section{REFERÊNCIAS}

[1] P. C. Avi, "Modelo Semi-empírico para a Modelagem da Transferência Simultânea de Calor e Água no Solo”. Dissertação de Mestrado, Unijui. Ijuí-RS, 2011.

[2] D. Hillel, "Environmental soil physics". London: Academic Press. 1998.

[3] M. N. Özisik, H. R. B. Orlande, "Inverse Heat Transfer: Fundamentals and Applications". Taylor Francis. New York, 2000.

[4] M. Van Genuchten, "A closed-form equation for predicting the hydraulic conductivity of unsaturated soils". Soil Science Society of America Journal, Madison, v. 44, p. 892-898, 1980.

[5] H. F. C. Velho, "Introdução aos problemas inversos: aplicações em pesquisa espacial". Instituto Nacional de Pesquisas Espaciais. São José dos Campos, SP, 2008. 